\title{
Tratamento de diálise em pacientes com insuficiência renal crônica
}

\author{
Treatment in dialysis patients with chronic renal failure
}

\author{
Gabriela Rocha Garcia Machado' \\ Fernanda Romanholi Pinhati ${ }^{2}$ \\ 1 Acadêmica do Curso de Medicina do Centro Universitário de Volta Redonda - UniFOA. \\ 2 Universidade do Estado do Rio de Janeiro - FAT/UERJ - Resende, RJ.
}

\section{Resumo}

A insuficiência renal crônica (IRC) é um estado sindrômico de perda progressiva e, geralmente, irreversível provocada por doenças que tornam o rim incapaz de realizar as suas funções. A instalação da doença caracteriza-se pelo declínio das funções bioquímicas e fisiológicas de todos os sistemas do organismo. Quando a função renal se reduz abaixo de $12 \%$ da capacidade normal é necessária a adoção de métodos de tratamento como, por exemplo, a diálise e o transplante renal. Por meio do diagnóstico, é possível instituir ao paciente um tratamento conversador ou a diálise, com o objetivo de adiar a piora da função renal, o aparecimento dos sintomas e prevenir as complicações associadas a essa doença. $O$ tratamento dialítico pode ser realizado por diálise peritoneal ou hemodiálise. A diálise peritoneal consiste na utilização de solução de diálise para a realização do processo de purificação. Enquanto a hemodiálise consiste em um processo de filtração dos líquidos extracorporais do sangue, através de uma máquina que substitui as funções renais. Ambas as formas de tratamento podem ocasionar complicações. A realidade da vida cotidiana dos doentes com IRC em hemodiálise é permeada de alterações físicas que impõem limitações ao cotidiano e exige adaptações. A IRC promove uma nova realidade de vida para o paciente, podendo impossibilitá-lo de organizar sua vida frente às possíveis mudanças que ocorrem na qualidade e no estilo de vida. Tais mudanças exigem que o paciente estabeleça estratégias de enfrentamento para aderir à nova condição de vida.

\section{Palavras-chave}

Insuficiência renal crônica, tratamento de diálise, qualidade de vida.

\begin{abstract}
The chronic renal failure (CRF) is a state syndromic progressive loss and usually irreversible caused by diseases that make the kidneys unable to perform their functions. The installation of the disease is characterized by the decline of biochemical and physiological functions of all body systems. When the renal function is reduced below $12 \%$ of normal capacity, the adoption of methods of treatment such as, for example, dialysis and renal transplantation. Through the diagnosis is possible to provide to the patient a conservative treatment or dialysis, in order to delay the worsening of renal function, the appearance of the symptoms and prevent complications associated with this disease. The dialysis can be performed by peritoneal dialysis or hemodialysis. The peritoneal dialysis uses the dialysis solution for carrying out the purification process. While hemodialysis is a process of filtration of liquids from extracorporeal blood by machine replacing renal function. The reality of everyday life of CRF patients on hemodialysis is fraught with physical changes that impose limitations to their daily life and demands adaptations. The CFR promotes a new reality to the patient's life, which can be impossible to adjust and manage, in the face of possible changes that take place in the life quality and style. Such changes require the patient to establish strategies to adhere to the new condition of life.
\end{abstract}

\section{Keywords}

Chronic renal failure, dialysis treatment, quality of life.

\section{Como você deve citar?}

MACHADO, Gabriela Rocha Garcia; PINHATI, Fernanda Romanholi. Tratamento de diálise em pacientes com insuficiência renal crônica. Cadernos UniFOA, Volta Redonda, n. 26, p. 137-148, dez. 2014. 


\section{INTRODUÇÃO}

A nefropatia crônica é uma condição mórbida decorrente de uma série de fatores, que possui como característica fisiopatológica básica a falência das funções renais, resultando no impedimento da eliminação eficaz de produtos residuais metabólicos do organismo, de forma a acarretar um progressivo acúmulo desses catabólicos no sangue (THOMASL e ALCHIERILL, 2005).

A falência das funções renais é caracterizada, na maioria dos casos, por nefropatia crônica, como insuficiência renal crônica (IRC). A IRC consiste em uma perda progressiva e irreversível das funções renais que pode se iniciar com um quadro agudo ou de maneira lenta e progressiva (TRENTINI et al., 2004).

A IRC não contempla uma cura. O tratamento definitivo indicado é o transplante renal, o qual é um processo demorado e, como alternativa para se manter a vida, opta-se pelo tratamento dialítico contínuo (SBN, 2012).

A diálise é um tratamento que visa repor as funções dos rins, retirando as substâncias tóxicas, o excesso de água e sais minerais do organismo, estabelecendo assim uma nova situação de equilíbrio (SBN, 2012). A diálise pode ser subdividida em duas modalidades: diálise peritoneal ou hemodiálise.

O objetivo do presente trabalho foi realizar uma revisão da literatura atual sobre o tratamento de diálise em pacientes com IRC, no intuito de abordar os benefícios e as alterações que o tratamento de diálise provocam na qualidade de vida do paciente.

Para o cumprimento deste trabalho foram usados artigos dos sites Scielo, BVS e Pubmed, além de livros da biblioteca UniFOA.

\section{REVISÃO DE LITERATURA}

\subsection{Diferenciação entre doença renal crônica (DRC) e insuficiência renal crônica (IRC)}

A expressão Doença Renal Crônica (DCR) foi recentemente definida pela Iniciativa de Qualidade em Desfechos de Doenças Renais da Fundação Nacional do Rim dos Estados Unidos (NKF - K/DOQI) como "a presença de dano renal ou diminuição da função renal por três ou mais meses" (ROMÃO Jr, 2004; THOMÉ et al., 2006).

DRC se refere a uma miríade de doenças, tanto sistêmicas que danificam os rins, quanto intrínsecas a esses órgãos, que se seguem à perda da função renal. Nessa doença resultante, o dano renal raramente é reparado, sendo que a perda da função renal persiste e compromete ainda mais o organismo.

A DRC abrange um espectro de problemas clínicos que vão desde anomalias detectáveis somente através de exames laboratoriais até o estágio tardio da doença, como a uremia (HARRISON et al., 2008). A doença apresenta-se assintomática até que o paciente tenha perdido cerca de $50 \%$ do funcionamento dos seus dois rins. Acima desse valor, vários sintomas podem ocorrer, como inchaço, pressão alta, anemia, entre outros (SBN, 2012).

Dentre as complicações mais frequentes da DRC estão os processos infecciosos, distúrbios do metabolismo ósseo e mineral, acidose metabólica, distúrbios do potássio, desequilíbrios da homeos- 
tase da água e do sódio, anemia, hipertensão arterial, alterações da nutrição, retardo do crescimento, insuficiência cardíaca e pericardite (LIMA, 2003).

Quando o rim deixa de realizar a maioria das suas funções, o estado clínico é denominado doença renal em estágio terminal (DRCT), sendo necessários transplantes ou diálises para a manutenção da vida. A natureza crônica e progressiva da DRC deve ser enfatizada, visto que o tratamento pode retardar ou bloquear a perda da função renal, com melhoria ou eliminação dos sintomas da uremia (HARRISON et al., 2008).

A insuficiência renal (IR) é uma doença sistêmica e consiste na via final comum de muitas diferentes doenças do rim e do trato urinário. A IR refere-se à situação na qual os rins não são mais capazes de remover os produtos de degradação metabólica do corpo ou de realizar suas funções reguladoras (SOUZA et al., 1996; SMELTZER et al., 2002), podendo se apresentar nas formas aguda (IRA) ou crônica.

A IRA consiste na redução rápida, em algumas horas, porém, temporária da função renal, uma vez que os rins podem voltar a funcionar após algumas semanas. A diminuição na função renal refere-se, principalmente, à redução do ritmo de filtração glomerular (RFG), todavia ocorrem também disfunções no controle do equilíbrio hidroeletrolítico e ácido-básico. Em muitas ocasiões o paciente precisa ser mantido com tratamento por diálise até que os rins voltem a funcionar (YU e ABENSUR, 2001).

A IRC refere-se a um diagnóstico sindrômico de perda progressiva e, geralmente irreversível da função renal de depuração, ou seja, da filtração glomerular (RFG) (RIBEIRO et al., 2008). Sua evolução pode ser lenta e progressiva. Essa perda resulta em processos adaptativos que, até certo ponto, mantêm o paciente sem sintomas da doença. Quando os rins possuem apenas 10 a $12 \%$ da função renal normal, pode-se tratar os pacientes com medicamentos e dieta. Contudo, quando a função renal se reduz abaixo dos valores citados, é imprescindível a adoção de outros métodos de tratamento, como, por exemplo, diálise ou transplante renal (SBN, 2012).

A IRC pode transpor quatro estágios: a) reserva renal reduzida (taxa de filtração glomerular [TGF] entre 35 a 50\% do normal); b) insuficiência renal reduzida (TGF entre 20 a 35\% do normal); c) falência renal (TGF entre 20 e $25 \%$ do normal); d) doença renal terminal (TGF abaixo de $20 \%$ do normal) (BOUNDY et al., 2004).

Os primeiros sintomas da IRC podem demorar anos para serem notados, o que demonstra grande capacidade adaptativa dos rins, permitindo que seres humanos mantenham-se vivos com apenas $10 \%$ da função renal (RIBEIRO et al., 2008).

A instalação da doença caracteriza-se pelo declínio das funções bioquímicas e fisiológicas de todos os sistemas do organismo, secundária ao acúmulo de catabólitos (toxinas urêmicas), alterações do equilíbrio hidroeletrolítico e ácido-básico, acidose metabólica, hipovolemia, hipercalemia, hiperfosfatemia, anemia e distúrbio hormonal, hiperparatireoidismo, infertilidade, retardo no crescimento, e outros (RIELLA, 2003).

Atualmente, a IRC apresenta-se como um sério problema de saúde pública em todo o mundo, sendo considerada uma epidemia de crescimento alarmante (MADEIRO et al., 2010). Segundo o Censo Brasileiro de Diálise, realizado em 2008, pela Sociedade Brasileira de Nefrologia, há, no Brasil, 684 Unidades Renais Cadastradas ativas na Sociedade Brasileira de Nefrologia (SBN, 2012), sendo que cerca de 327 dessas unidades que responderam ao questionário, 288 (93,8\%) tinham convênio com o Sistema Único de Saúde (SUS) e 19 (6,2\%) com empresas de seguro saúde privado. 0 número de pacientes avaliados em diálise foi de 87.044 , considerando que $57,4 \%$ destes encontravam-se na região sudeste. 
O número de pacientes que se encontram em tratamento dialítico tem crescido anualmente. Comparado com o ano anterior, o aumento anual do número de pacientes foi de $10,1 \%$, em $2005 ; 8,8 \%$, em 2006 e $3,9 \%$, em 2007. O crescimento mais marcante foi o estimado para o ano de $2008,18,3 \%$ (SESSO et al., 2008).

De acordo com o Censo Brasileiro de Diálise, realizado em 2011, estavam cadastradas na SBN 687 unidades em julho de 2011. Destas, 643 tinham programa ativo para tratamento dialítico crônico e $353(54,9 \%)$ responderam ao questionário e tiveram seus dados analisados. Entre as 353 unidades, $91,5 \%$ tinham convênio com o SUS e $75,6 \%$ com outros convênios de saúde suplementar. 0 número de pacientes dialíticos vem aumentando gradualmente ao longo dos anos de 42.695, em 2000, para 91.314 , em 2011, tendo permanecido constante em relação a 2010. Mais da metade desses pacientes encontrava-se na região Sudeste (SESSO et al., 2012).

O número estimado de pacientes que iniciaram tratamento, em 2011, no Brasil, foi de 28.680 . Essas estimativas são consideravelmente maiores que as observadas em 2010, quando 18.972 pacientes novos iniciaram o tratamento. Todos os valores referentes à taxa de incidência anual estimada de pacientes em diálise, no Brasil, por região, de acordo com o censo de 2011, estão apresentados no Gráfico 1 abaixo (SESSO et al., 2012).

Gráfico 1 - Incidência estimada de pacientes em diálise no Brasil, por região, 2011.

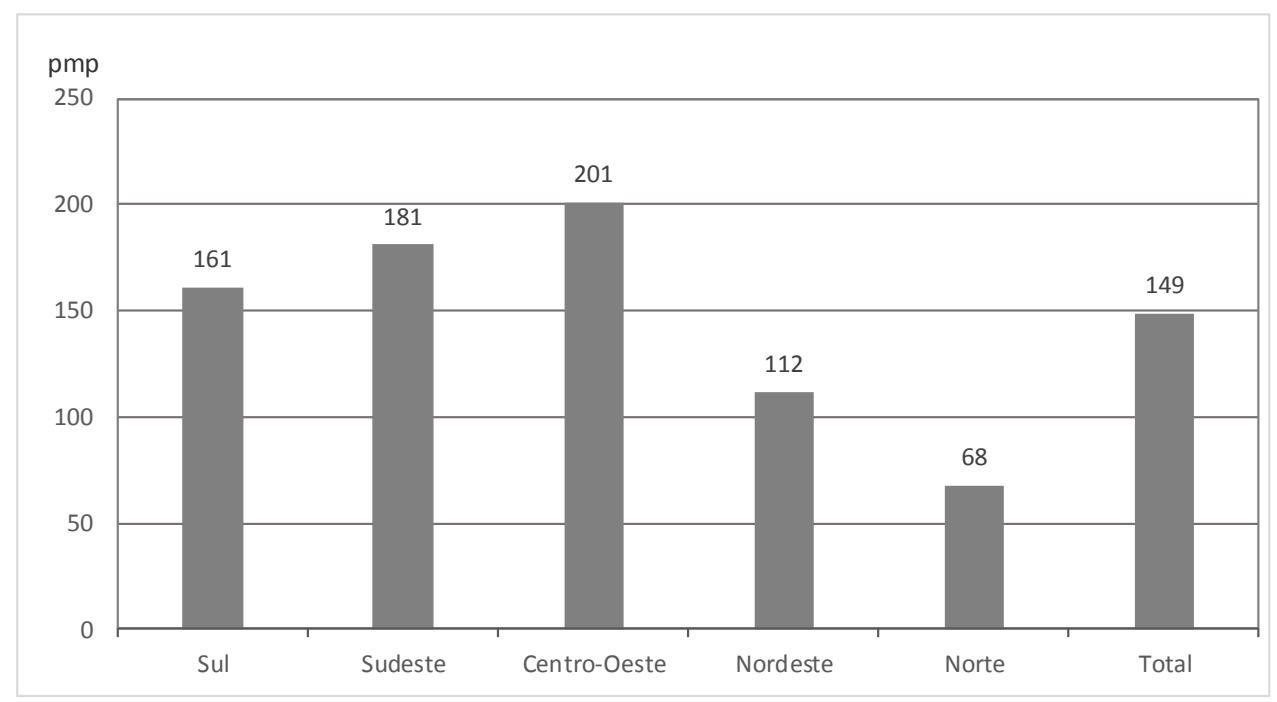

(SESSO, et al., 2012), pmp (pacientes por milhão da população).

Comparando com a incidência anual estimada de paciente em diálise no Brasil por região, entre os censos de 2008 e 2011 , nota-se uma diminuição da incidência nas regiões norte, nordeste e centro-oeste, sendo que, no norte, houve uma redução de $46 \%$.

\subsection{Tipos de tratamento de diálise}

Ao ser diagnosticada a IRC, deve-se instituir ao paciente um tratamento conservador ou dialítico 0 mais precoce possível, pois as ocorrências de complicações podem levar à morte (BARBOSA et al., 2006).

Algumas medidas terapêuticas conversadoras podem ser propostas como: tratamento dietético, medicamentoso, controle da pressão arterial e da glicemia, e modificações no estilo de vida. Tais medidas são utilizadas para retardar a piora da função renal, reduzir os sintomas e prevenir complica- 
ções associadas à DRC. 0 tratamento conversador é admitido ao se diagnosticar a DRC, podendo ser mantido a longo prazo, visando a um impacto positivo na sobrevida e qualidade de vida dos pacientes (ROMÃO Jr, 1995; SBN, 2012). Quanto mais precoce se começar o tratamento conversador maiores são as chances para preservar a função dos rins por mais tempo.

A recomendação do programa dialítico ocorre quando o tratamento conservador não é capaz de manter a qualidade de vida do paciente e quando há o surgimento de sinais e sintomas importantes da uremia (THOMÉ et al., 2006), podendo ser realizado através de diálise peritoneal ou a hemodiálise.

A hemodiálise é o tratamento dialítico mais empregado, que deve ser realizado pelos clientes portadores de IRC por toda vida ou até se submeterem a um transplante renal bem-sucedido. Assim, para garantir a eficácia da hemodiálise é necessário que os pacientes manifestem a adesão ao tratamento dialítico (MADEIRO et al., 2010).

A hemodiálise consiste em um processo de filtração dos líquidos extracorporais do sangue realizado por uma máquina denominada dialisador, que substitui as funções renais (CAVALCANTE et al., 2011). Para iniciar o tratamento, é preciso instituir, cirurgicamente, uma fístula arteriovenosa ou a colocação de um cateter específico na veia, para ter acesso à circulação do paciente (LOMBA e LOMBA, 2005; HOSPITAL ALBERT EINSTEIN, 2012). Durante a hemodiálise, parte do sangue do corpo do paciente é retirado pela fístula ou cateter específico, sendo conduzido através da linha arterial do dialisador, onde é filtrado, retornando ao paciente pela linha venosa (SAÚDE BIAZI, 2012).

Geralmente, a hemodiálise é realizada em sessões com duração média de três a quatro horas, três vezes por semana. Podem existir modificações no tempo e na frequência dessas sessões de acordo com o estado clínico do paciente (SBN, 2012; SAÚDE BIAZI, 2012).

Durante as sessões podem surgir efeitos colaterais causados por rápidas alterações do volume de líquido e no equilíbrio químico do organismo do paciente. Os efeitos mais comuns são as cãibras musculares e a hipotensão, sendo que esta última pode deixar o paciente fraco, atordoado e com náuseas. Tais efeitos podem ser evitados com uso de medicamentos e seguindo uma dieta prescrita pelo médico. Entretanto, o paciente requer meses para se adequar ao tratamento hemodialítico (HOSPITAL ALBERT EINSTEIN, 2012).

O principal objetivo do tratamento hemolítico é minimizar os sintomas causados pelo mau funcionamento dos rins e beneficiar ao paciente uma melhor qualidade de vida, permitindo-o se sentir bem, considerando-se sempre o monitoramento dos níveis plasmáticos de potássio, ureia, sódio e cloretos (SAÚDE BIAZI, 2012).

Outro tipo de tratamento que substitui as funções dos rins e tem o mesmo objetivo da hemodiálise é a diálise peritoneal (DP). Para a realização da filtração do sangue, esse tipo de diálise utiliza-se do peritônio, membrana localizada dentro do abdômen que reveste os órgãos internos. A membrana peritoneal, que age como um "dialisador", é, na verdade, uma membrana semipermeável, heterogênea, contendo múltiplos e diferentes poros, com uma fisiologia e uma anatomia relativamente complexa (DAUGIRDAS et al., 2003). A DP consiste na utilização de solução de diálise, também chamada de "banho" de diálise, para a realização do processo de purificação. A solução de diálise passa da bolsa de plástico através do cateter para a cavidade abdominal, onde permanece por várias horas. A solução é então drenada e uma nova solução volta a preencher o abdômen, recomeçando o processo de depuração. Em geral, são feitas de 3 a 6 trocas de líquido durante o dia. Cada momento de troca entre as bolsas de solução de diálise deve ser executado cuidadosamente para que se evitem infecções (SAÚDE BIAZI, 2012). 
Esse tipo de diálise é comumente a primeira escolha para o tratamento da DRC, principalmente em crianças e adolescentes. A DP está indicada por oferecer vantagens ao paciente, especialmente por possibilitar melhor controle bioquímico da uremia, da anemia e hipertensão arterial, permitindo a preservação da função renal residual, a nutrição e ingestão de líquidos com menor restrição, demandando menor necessidade de transfusões sanguíneas e ausência da síndrome do desequilíbrio per diálise, à qual os pacientes em hemodiálise estão sujeitos (MOREIRA et al., 1996).

A DP pode trazer riscos ao paciente, caso não sejam respeitados alguns requisitos indispensáveis ao sucesso da mesma, como condições minimamente adequadas de moradia, antissepsia do ambiente reservado à DP, motivação e domínio da técnica por parte dos familiares, que são responsáveis ou ajudam no processo, dentre outros (MOREIRA et al., 1996).

A principal contraindicação à DP é a presença de lesão do peritônio causada por fibrose ou doença maligna. A principal razão para a interrupção de uma diálise peritoneal é a ocorrência de episódios frequentes de peritonite, embora a desistência dos pacientes e família também seja um fator (DAUGIRDAS, 2003).

Assim como a hemodiálise, a diálise peritoneal pode ocasionar complicações, sendo a principal a peritonite, que é a infecção do peritônio. Na maioria das vezes, o início da infecção se dá no orifício de saída cutânea (IOS) do cateter peritoneal ou do túnel subcutâneo. Essas infecções são causas importantes de morbidade e, com frequência, requerem a retirada do cateter, podendo inviabilizar o tratamento (LENTINO e LEEHEY, 2003). A peritonite deve ser tratada de forma rápida e corretamente. Outra complicação comum é o entupimento do cateter, que causa dificuldade na drenagem do líquido no abdômen. $O$ desentupimento deve ser feito no centro de diálise, onde o paciente faz o acompanhamento (MOREIRA et al., 1996; SAÚDE BIAZI, 2012).

Outra importante complicação da DP que, ocasionalmente, foi citada nas literaturas, é a prevalência da Doença Óssea Adinâmica (DOA). A DOA tem sido detectada em alta porcentagem de pacientes, podendo chegar a $61 \%$ dos pacientes durante essa modalidade dialítica (SANTANA, et al., 2002).

Existem três tipos de diálise peritoneal: diálise peritoneal ambulatorial contínua (DPAC), a diálise peritoneal cíclica contínua (DPCC) e diálise peritoneal intermitente (DPI).

A DPAC representa um regime contínuo de DP manual em que a solução de diálise está continuamente presente na cavidade abdominal durante 7 dias da semana (TWARDOWSKI, 1992). É a forma mais comum de diálise peritoneal, sendo indicada, principalmente, para pacientes com dificuldade de ultrafiltração por alta permeabilidade determinada pelo PET (do inglês Peritoneal Equilibrium Test), pacientes idosos, crianças e aqueles para os quais a hemodiálise não é conveniente ou possível (RIELLA, 2003).

Esse método, geralmente, é realizado em casa e independe do equipamento. 0 próprio paciente pode fazer a infusão e a retirada (drenagem) da solução de diálise do abdômen ou pode ser auxiliado por outra pessoa treinada para fazer essas trocas de bolsas, eliminando assim a necessidade de acesso venoso (MOREIRA et al., 1996; SAÚDE BIAZI, 2012).

Nesse tipo de diálise peritoneal, a solução fica no abdômen durante 4 horas. 0 processo de drenar o dialisado e substituí-lo por uma solução nova leva de 30 a 40 minutos. Essa pequena interrupção de tempo ao longo das 4 a 5 trocas diárias não desqualifica o regime de continuidade, desde que não exceda $10 \%$ do total do tempo necessário à diálise (TWARDOWSKI, 1992; SAÚDE BIAZI, 2012). 
A DPCC, também chamada de diálise peritoneal automatizada (DPA), é similar à DPAC, todavia nesta há a conexão do cateter a uma máquina (cicladora) que preenche o abdômen e drena a solução de diálise automaticamente. Geralmente é realizada à noite, enquanto o paciente dorme, o que permite maior liberdade ao mesmo durante o dia. Em função dessa característica, a DPA foi rapidamente expandida como uma resposta às demandas de diálise e melhor qualidade de vida (DIAZ-BUXO, 1999). Esse método é pouco utilizado no Brasil devido ao alto custo da máquina necessária (SAÚDE BIAZI, 2012).

A DPA vem tomando espaço entre as opções de diálise para os pacientes renais crônicos ou agudos por sua segurança, menores índices de infecções, menor quantidade de tempo dedicado à técnica diariamente, maior flexibilidade para a prescrição de diálise para atingir adequação, melhor controle da ultrafiltração das sessões dialíticas (proporcionada pelos registros eletrônicos da mesma) e pela melhor qualidade de vida do paciente que utiliza esse método dialítico (ILHA e PROENÇA, 1999).

O terceiro tipo de diálise peritoneal, a DPI, também necessita de uma máquina para a infusão e drenagem da solução de diálise. Esse instrumento pode ser do mesmo tipo ao empregado na DPAC.

A DPI é, normalmente, realizada em hospital, sendo indicada para aqueles pacientes que não podem realizar outras formas de tratamento de diálise, como paciente com alta permeabilidade de membrana e função renal residual significativa. Esse método está associado a um maior número de complicações (D'ÁVILA et al., 1999; SAÚDE BIAZI, 2012).

0 tratamento com a DPI perdura por cerca de 24 horas, em ambiente hospitalar, com trocas a cada 1-2 horas, duas vezes por semana (40 a 60 litros). No período entre as diálises, o abdômen seco permanece seco (DAUGIRDAS, 2003; RIELLA, 2003).

Apesar de existirem diferentes tipos de diálise, todos os métodos são importantes e precisam de um acompanhamento médico rigoroso. Entretanto, de acordo com as condições clínicas de cada paciente, a preferência por um ou outro método será decidida pelo médico em conjunto com o paciente e sua família, de acordo com o quadro clínico e estilo de vida.

\subsection{Qualidade de vida durante a diálise}

A qualidade de vida é um conceito amplo e complexo. Refere-se a aspectos subjetivos percebidos de diferentes modos, contemplando as esferas de bem-estar físico, mental, desempenho no trabalho e participação social. Portanto, a definição de qualidade de vida depende da perspectiva que se deseja abordar, visto que é multidimensional (MARTINS et al., 1996; FLANAGAN, 1982).

A IRC, como doença crônico-degenerativa, surge na vida de uma pessoa promovendo uma nova realidade. Essa realidade guarda, em sua essência, outras infinitas possibilidades de transformação, que se apresentam de maneiras formais, reais, concretas ou abstratas, desde que encontrarem condições propícias determinantes. As possibilidades que se apresentam no decorrer do adoecer, transformando o indivíduo de acordo com sua realidade, podem impossibilitá-lo a se acostumar ou organizar sua vida frente a cada possibilidade de uma nova realidade (MACHADO e CAR, 2003). Dentre as possíveis mudanças que podem ocorrer na qualidade e no estilo de vida de um paciente com IRC, pode-se citar: a diminuição da energia física, alteração da aparência pessoal e novas incumbências. Tais fatores exigem que o paciente estabeleça estratégias de enfrentamento para aderir à nova condição de vida (MADEIRO et al., 2010).

Considerando os benefícios do tratamento dialítico, prolongar a vida dos pacientes com DRC, as condições impostas pela doença e pelo próprio tratamento, provocam uma série de alterações 
sistêmicas, metabólicas e hormonais, que podem afetar adversamente a condição nutricional desses pacientes (KAMIMURA et al., 2008).

Estudos indicam que indivíduos sujeitos à diálise enfrentam perdas e alterações estressantes da imagem e das funções orgânicas. Como consequência dessas perdas, muitas pessoas submetidas à diálise tornam-se deprimidas e ansiosas (FERNANDES, 1998). De modo geral, os problemas psicológicos e sociais consequentes da IRC e do tratamento reduzem, quando os programas de diálise incentivam o indivíduo a ser independente e a retomar seus interesses anteriores (MADEIRO et al., 2010).

A realidade da vida cotidiana dos doentes com IRC em hemodiálise é permeada de alterações físicas que impõem limitações ao cotidiano e exige adaptações. No ir-e-vir da realidade da vida cotidiana desses doentes, o dia seguinte emerge como uma pausa de alívio nas alterações físicas e pequenas atividades laboriosas podem até ser realizadas. Aos doentes do sexo feminino, as atividades domésticas oferecem oportunidade de ocupação do tempo e a percepção da capacidade individual como ser humano produtivo. Entretanto, aos do sexo masculino, o dia seguinte é percebido apenas pela melhora das condições físicas, já não exercem atividade ou esforço físico que possam considerar como "trabalho" (MACHADO e CAR, 2003).

Alguns estudos concluíram que há o comprometimento da capacidade funcional das pessoas que fazem hemodiálise, dificultando a atividade laboral, sobretudo daquelas na faixa etária entre 18 e 59 anos, considerada economicamente ativa na sociedade (LARA e SARQUIS, 2004). Pessoas em tratamento hemodialítico têm dificuldades para trabalhar em razão do comprometimento físico, tempo destinado à hemodiálise e dificuldades de contratação após início do tratamento (BERTOLIN, 2007). Tal situação provoca diminuição da autoestima e os pacientes passam a ser dependentes financeiramente $\mathrm{e}$ a necessitar, na maioria das vezes, do auxílio de um familiar ou pessoa próxima (KIRCHNER et al., 2011).

A qualidade de vida das pessoas em tratamento hemodialítico possui uma relação positiva entre os aspectos emocionais e de escolaridade, sugerindo que pessoas com maior escolaridade tenham recursos intelectuais capazes de gerar melhor adaptação emocional às consequências da IRC e de tratamento hemodialítico (CASTRO et al., 2003).

O tempo de tratamento é importante no agravamento de comorbidades, e estas têm sido apontadas em vários estudos, como sendo determinantes na sobrevida de clientes em tratamento hemodialítico (MORSCH et al., 2005).

Destaca-se que as mudanças de comportamento a que são submetidos os pacientes, refletem diretamente no comprometimento da qualidade de vida. E a forma como reagem frente a essas mudanças e os mecanismos que utilizam para enfrentá-las estão relacionados ao apoio recebido de seus entes queridos, além de suas crenças e valores (LIMA e GUALDA, 2000).

As dificuldades de adesão ao tratamento hemodialítico relatadas pelos pacientes deve-se às possíveis fontes de estresse que surgem durante o tratamento como: isolamento social, perda do emprego, dependência da Previdência Social, parcial impossibilidade de locomoção e passeios, diminuição da atividade física, necessidade de adaptação à perda da autonomia, alterações da imagem corporal e, ainda, um sentimento ambíguo entre medo viver e de morrer (MACHADO e CAR, 2003). Com tantos agravantes, o paciente torna-se insatisfeito consigo mesmo, sentindo-se excluído (CAVALCANTE et al., 2011).

As reações apresentadas pelos pacientes com IRC influenciam no seu meio social, cultural, em suas crenças e nos seus valores pessoais. 0 apoio individual, grupal e o suporte das relações sociais, 
de trabalho e de familiares podem ser benéficos como estratégias de enfrentamento (TRENTINI et al., 1990). 0 suporte social pode servir como amparo emocional nas consequências negativas durante o decaimento da função física no processo de adoecer (KIRCHNER et al., 2011).

A relação de dependência por cuidados pode ser frustrante para o paciente portador de IRC. Muitas vezes, precisa modificar suas responsabilidades e as funções estabelecidas, visando favorecer a adesão à hemodiálise, podendo gerar estresse e sentimentos de culpa e incapacidade no paciente.

A família, vizinhos e amigos podem ajudar no enfrentamento da doença e de suas consequências, uma vez que integram o contexto no qual o indivíduo está inserido, assumindo funções de proteção e socialização. A família, como uma unidade, desenvolve um sistema de valores, crenças e atitudes face à saúde e doença, que são expressas e demonstradas por meio dos comportamentos de saúde-doença de seus membros (MADEIRO et al., 2010). Muitas vezes, a experiência do adoecimento leva ao fortalecimento das relações sociais, principalmente, familiares. Destaca-se que o apoio social recebido de familiares e amigos se traduzem em qualidade do suporte emocional nas relações sociais e interfere na saúde das pessoas (RUDNICKI, 2007).

A pessoa com IRC vivencia intranquilidade em seu estado de saúde e vê-se em constante perigo de perder a integridade, tanto física como psíquica, ou sua posição na família e na sociedade, em decorrência das alterações em suas funções orgânicas (CICONELLI, 1981). 0 atendimento psicológico proporciona mais qualidade de vida para essas pessoas, auxilia no rompimento de tabus e preconceitos, além de incentivá-las a desenvolver suas capacidades, levando-as a verem a doença de outra forma, proporcionando um atendimento integral, com melhora da qualidade de vida dessas pessoas (RESENDE et al., 2007).

Dessa forma, é fundamental o apoio dos profissionais de saúde para que o indivíduo em tratamento e sua família possam assimilar e responder melhor à vivência da doença crônica.

\section{CONCLUSÕES}

Em resumo, a qualidade de vida de um paciente com IRC é drasticamente afetada após o seu diagnóstico e início do tratamento. 0 apoio familiar e dos profissionais de saúde têm demonstrado ser fundamentais para garantir a adesão do paciente ao tratamento e proporcionar um mínimo de conforto a esse paciente. Nesse sentido, reflexões sobre os fatores que mais interferem na qualidade de vida dessas pessoas permitem a promoção de um cuidado mais planejado e integral a ser prestado pela equipe de saúde, permitindo um viver com mais qualidade. 


\section{REFERÊNCIAS}

ABRAHÃO, Sarah Silva. Determinantes de falhas da diálise peritoneal no domicílio de crianças e adolescentes assistidos pelo hospital das clínicas da UFMG. 2006. Dissertação (Mestrado em Ciências da Saúde Área de Concentração em Saúde da Criança e do Adolescente), Universidade Federal de Minas Gerais, Belo Horizonte, 2006.

BERTOLIN, Daniela Comelis. Modos de enfrentamento de pessoas com insuficiência renal crônica terminal em tratamento hemodialítico. 2007. Dissertação (Mestrado em Enfermagem). Escola de Enfermagem de Ribeirão Preto da Universidade de São Paulo, Ribeirão Preto, São Paulo, 2007.

BOUNDY, J. Enfermagem Médico-Cirúrgica. Tradução Carlos Henrique Cosendey. Rio de Janeiro: Reichman \& Afonso Editores; 2004.

CASTRO, M. et al. Qualidade de vida de pacientes com insuficiência renal crônica em hemodiálise avaliada através do instrumento genérico SF- 36. Revista de Associação Médica Brasileira, v. 49, p. 245-249, 2003.

CAVALCANTE, F. A. et al. O uso lúdico em hemodiálise: buscando novas perspectivas na qualidade de atendimento ao paciente no centro de diálise. Revista Eletrônica da Facimed, v. 3, p. 371-384, 2011.

CICONELLI, Maria Ignez Ribeiro de Oliveira. 0 paciente com insuficiência renal crônica em hemodiálise: descrição do tratamento e problemas enfrentados pelo paciente, sua família e equipe de saúde, 1981. Dissertação (Mestrado em Enfermagem). Escola de Enfermagem de Ribeirão Preto da Universidade de São Paulo, Ribeirão Preto, São Paulo, 1981.

DAUGIRDAS, J.T.; BLAKE, P.G.; Ing, T.S. Manual de Diálise. 3a. ed. Rio de Janeiro: Editora Médica e Científica LTDA, 2003.

DIAZ-BUXO, J.A. Automated peritoneal dialysis. A therapy in evolution. In: RONCO, C. AMICI, G. FERIANI, M. and VIRGA, G. (Eds). Automated Peritoneal Dialysis. Basel: Karger, p.1-14, 1999.

FERNANDES, M.G.M, et al. Diagnósticos de Enfermagem de uma família com um membro portador de IRC. Revista de Enfermagem, v.4, p.18-24, 1998.

FLANAGAN, J.C. Measurement of qualit of life: current state of the art. Archives of Physical Medicine and Rehabilitation, v. 63, p. 56-59, 1982.

HARRISON, T.R. et al. Harrison medicina interna. 17. ed. Rio de Janeiro: McGraw-Hill, 2008.

HOSPITAL ALBERT EINSTEIN (Brasil). Centro de diálise. Tratamentos. Diálise Peritoneal. Hemodiálise. São Paulo, 2012. Disponível em: <http://www.einstein.br/Hospital/centro-de-dialise/Paginas/centrode-dialise.aspx>. Acesso em 10 out. 2012.

ILHA, L.H.; PROENÇA, M.C. Diálise Peritoneal. In: BARROS, E. MANFRO, R.C. THOMÉ, L.F.S. GONÇALVES, L.F.S. [et al.]. Insuficiência Renal Crônica. Nefrologia, Rotinas, Diagnósticos e Tratamentos. $2^{\mathrm{a}}$ ed. Porto Alegre: Artes Médicas Sul Ltda, 1999.

KAMIMURA, M. A. et al. Gasto energético de repouso em pacientes com doença renal crônica. Revista Brasileira de Nutrição. v. 1, p.75-84, 2008. 
KIRCHNER, R. M. et al. Análise do estilo de vida de renais crônicos em hemodiálise. 0 Mundo da Saúde. v. 35, p. 415-421, 2011.

LARA, E. A.; SARQUIS, L. M. M. O paciente renal crônico e sua relação com o trabalho. Cogitare Enfermagem. v. 9, p. 99-106, 2004.

LENTINO, J. R. \& LEEHEY, D. J. Infecções. In: DAUGIRDAS, J. T.; BLAKE, P.G.; Ing, T.S. Manual de Diálise. $3^{\mathrm{a}}$ ed. Rio de Janeiro: Medsi, cap. 28, p. 509-534, 2003.

LIMA, A. F. C; GUALDA, D. M. R. Reflexão sobre a qualidade de vida do cliente renal crônico submetido à hemodiálise. Nursing. v. 3, p.20-23, 2000.

LIMA, E.M. Complicações da Insuficiência Renal Crônica. In: SILVA, A.C.S. Manual de Urgências em Pediatria. Rio de Janeiro: Medsi, p.324-339, 2003.

LOMBA, A.; LOMBA, M. Especialidades Médicas. 2ed. Olinda: Objetivo saúde, p. 114-116, 2005.

MACHADO, L. R. C.; CAR, M. R. A dialética da vida cotidiana de doentes com insuficiência renal crônica: entre o inevitável e o casual. Revista da Escola de Enfermagem - USP. v. 3, p. 27-35, 2003.

MADEIRO, A. C. et al. Adesão de portadores de insuficiência renal crônica ao tratamento de hemodiálise. Acta Paulista de Enfermagem, v.23, p. 546-551, 2010.

MARTINS, L. M.; FRANÇA, A. P. D.; KIMURA, M. Qualidade de vida de pessoas com doença crônica. Revista Latino-Americana de Enfermagem. v. 4, p. 5-8, 1996.

MOREIRA, P. R. R. et al. Infecção do orifício de saída: uma complicação importante na diálise peritoneal ambulatorial contínua. Jornal Brasileiro de Nefrologia, v. 18, p. 348-355, 1996.

MORSCH, C.; GONÇALVES, L. F., BARROS, E. Índice de gravidade da doença renal, indicadores assistenciais e mortalidade em pacientes em hemodiálise. Revista de Associação Médica Brasileira, v. 51, p. 296$300,2005$.

RESENDE, M. C. et al. Atendimento psicológico a pacientes com insuficiência renal crônica: em busca de ajustamento psicológico. Psicologia Clínica. v. 19, n. 2, p. 87-99, 2007.

RIBEIRO, R. C. H. M. et al. Caracterização e etiologia da insuficiência renal crônica em unidade de nefrologia do interior do Estado de São Paulo. Acta Paulista de Enfermagem v.31, p. 207-211, 2008.

RIELLA, M.C. Princípios de Nefrologia e Distúrbios Hidroeletrolíticos. 4 ed. Rio de Janeiro: Guanabara Koogan; cap. 36, p. 649-60, 2003.

ROMÃO, J. J. E. Doença Renal Crônica: Definição, Epidemiologia e Classificação. Jornal Brasileiro de Nefrologia, v. 26, p. 1-3, 2004.

ROMÃO Jr, J. E. Insuficiência renal crônica. In: CRUZ, J,; PRAXEDES, J.N. Nefrologia. São Paulo: Sarvier; cap. 17, p.187-200, 1995.

RUDNICKI, T. Preditores de qualidade de vida em pacientes renais crônicos. Estudo de Psicologia Campinas. v. 24, n.3, p.343-351, 2007. 
SANTANA, A. P. S. et al. Osteodistrofia renal em pacientes submetidos à diálise peritoneal ambulatorial contínua. Jornal Brasileiro de Nefrologia, v.24, p.97-102, 2002.

SAÚDE BIAZI (Brasil). Diálise e Hemodiálise. São Paulo, 2012. Disponível em: <http://biazi.br.tripod. com/saudebiazi/id14.html>. Acesso em 22 out. 2012.

SESSO, R. et al. Relatório do Censo Brasileiro de Diálise, 2008. Jornal Brasileiro de Nefrologia, v. 30, p. 233-238, 2008.

SESSO, R. C. C. et al. Diálise Crônica no Brasil - Relatório do Censo Brasileiro de Diálise, 2011. Jornal Brasileiro de Nefrologia, v.34, p.272-277, 2012.

BARBOSA, D. A. et al. Co-morbidade e mortalidade de pacientes em início de diálise. Acta Paulista de Enfermagem, v.19, p. 304-309, 2006.

SMELTZER, S. C.; BARE, B. G. Brunner \& Suddarth: tratado de enfermagem médico-cirúrgica. v. 3, 9ed. Rio de Janeiro: Guanabara Koogan; 2002.

SOCIEDADE BRASILEIRA DE NEFROLOGIA (Brasil). Doenças comuns. Tratamento. São Paulo, 2012. Disponível em: <http://www.sbn.org.br/>. Acesso em 16 out. 2012.

SOUZA, A. G. M. R.; MANSUR, A. J. Sociedade de Cardiologia do Estado de São Paulo (SOCESP) 2ed. São Paulo: Atheneu; p. 332, 1996.

TRENTINI, M.; SILVA, D. G. V.; LEIMANN, A. H. Mudanças no estilo de vida enfrentadas por pacientes em condições crônicas de saúde. Revista Gaúcha de Enfermagem. v. 11, p. 18-28, 1990.

THOMASL, T. V.; ALCHIERILL, J. C. Qualidade de vida, depressão e características de personalidade em pacientes submetidos à Hemodiálise. Avaliação Psicológica, v. 4, p. 57-64, 2005.

THOMÉ, F. S. et al. Doença renal crônica. In: BARROS, E. et al. Nefrologia: rotinas, diagnóstico e tratamento. 3a ed. Porto Alegre: Artmed. cap. 24, p. 381-404, 2007.

TRENTINI, M. et al. Qualidade de vida de pessoas dependentes de hemodiálise considerando alguns aspectos físicos, sociais e emocionais. Texto e Contexto Enfermagem, v.13, p. 74-82, 2004.

TWARDOWSKI, Z. J. Nightly peritoneal dialysis. Why, who, how and when? ASAIO Trans v. 36, p. 8-16, 1990.

YU, L.; ABENSUR, H. Insuficiência renal aguda. Conceito, diagnóstico, prevenção e tratamento da insuficiência renal aguda. São Paulo: SBN; 2012 [citado 15 jun 2007]. Disponível em: <www.sbn.org. br/Diretrizes/ira.htm>. 